\title{
This issue of Biophysical Reviews is a little different
}

\author{
Cristobal G dos Remedios ${ }^{1}$
}

Received: 26 March 2016 / Accepted: 29 March 2016/Published online: 26 April 2016

(C) International Union for Pure and Applied Biophysics (IUPAB) and Springer-Verlag Berlin Heidelberg 2016

\section{Letters to the Editor}

Two Letters-to-the-Editor follow the careers paths of biophysicists into biomedical science. The first is written by a very successful medical graduate who, soon after graduating, decided to explore a career in "Big Pharma". In it he outlines his thinking and the decisions he made as he progressed through to this mid-stage of his career. His advice will probably resonate with young biomedical scientists contemplating a similar career path.

The second Letter-to-the-Editor is from an eminent biophysicist who began his career as a chemistry graduate. At an early stage of his career he decided that biological chemistry, particularly protein chemistry, was to be his future. Unlike the above Letter, he writes from the perspective of a scientist who has reached his personal goals and is now focussed on turning his advances in science and technology into commercial reality.

\section{How to turn proteins into highly specific and sensitive biosensors}

In this issue, Tomonobu M. Watanabe and his colleagues at Riken in Osaka take the reader on a guided "tour" of how they and others have designed and

Cristobal G dos Remedios

crisdos@anatomy.usyd.edu.au

1 The University of Sydney, Sydney NSW 2006, Australia refined the development of genetically engineered (GE) fluorescent protein sensors that can monitor a surprising range of biophysical responses.

Did you ever wonder how we learned to genetically engineered proteins to become fluorescent, and then to fine-tune their fluorescence excitation and emission spectra to specifically monitor a wide range of chemical conditions in the complex mixtures such as found in living cytoplasm? These include $\mathrm{pH}$, chloride, and calcium ions $(\mathrm{pCa})$ monitors. By developing GE proteins with selectable absorption and emission spectra, Foster distances can monitor conformational changes in GE protein sensors over lengthy periods with little loss of sensitivity. These specific and stable monitors can be introduced into living cells to enable measurements to be made with millisecond resolution and with a sensitivity that can be fine-tuned.

They describe the strategies that underlie the development of a wide range of molecular functions including biosensors for Redox reactions (involved in a wide range of processes such as regulating transcription, oxidative phosphorylation), monitors of temperature and pressure, and molecular crowding. Watanabe and his colleagues conclude by sharing their vision for future sensors such as protein proximity sensing.

I found this review inspirational. Imagine what cardiologists could do it they could put a strain gauge inside cultured cardiomyocytes from failing and nonfailing and healthy human hearts. It requires only a modest stretch of the imagination to consider monitoring calcium ion and tension transients in real time in biopsies of failing and non-failing donor human hearts in culture. We might even solve the mystery of the molecular basis of the elusive Frank-Starling law of the heart. 


\section{Biophysics of tyrosine}

A two-part review addresses the question of how to go about probing structural changes in proteins without attaching bulky extrinsic fluorescent probes. Many of us are used to the notion of using tryptophan fluorescence because Trp is present in most proteins, and because it has accessible fluorescence properties (high quantum yield, fluorescence polarization, and it is sensitive to molecular environment). Probing tyrosine residues in proteins always seemed to me to be more challenging. I was wrong.

The two-part review by Jan M. Antosiewicz and the venerable David Shugar made me think again. Part I of the review takes the reader through the biophysics of spectroscopies including: conventional UV absorption, UV difference, and second-derivative spectroscopy, fluorescence and FRET spectroscopy, linear and circular dichroism, and Raman spectroscopy. If you are an experienced spectroscopist, then skip to Part 2, which describes how these spectroscopies can be used to follow individual Tyr residues on the surface of proteins and/or buried deep within them. It is both rigorous and an eye-opener.

\section{The next two issues are special issues}

The idea behind Special Issues in biophysics it to assemble a group of expert reviewers that address different aspects of a focussed topic in biophysics. Readers can benefit because they are presented with a collection of related reviews on the selected topic. Authors also benefit because the Springer-Nature statistics tell us that these authors are more likely to get cited.

The next issue (volume 8,\# 3) is edited by Special Editor Laura Finzi and Executive Editor Wilma Olson. It is devoted to the inter-relationship of DNA supercoiling, protein interactions, and gene function.

The final issue for the year (volume 8 \#4) is edited by Damien Hall, one of our Executive Editors. It is designed to honor Prof. Donald J. Winzor on the occasion of his 80th birthday. His illustrious career has influenced many former students and colleagues, and this is likely to be one of the largest issues ever produced of Biophysical Reviews.

Finally, many contributors have asked for Biophysical Reviews to be listed in PubMed and Medline. An application that was submitted 2 years ago failed, but this time, with the letters of support from two Nobel Prize winners, and two others, we understand this listing will commence in time for the above two Special Issues.

Cris dos Remedios

Editor-in-Chief.

March 25, 2016. 